\title{
The effectiveness and limitations of regulatory warnings for the safe prescribing of citalopram
}

This article was published in the following Dove Press journal:

Drug, Healthcare and Patient Safety

19 August 2015

Number of times this article has been viewed

\section{Kevin J Friesen \\ Shawn C Bugden}

Faculty of Health Sciences, College of Pharmacy, University of Manitoba, Winnipeg, MB, Canada
Correspondence: Shawn C Bugden Faculty of Health Sciences, College of Pharmacy, University of Manitoba, 750 McDermot Avenue, Winnipeg, Manitoba R3E 0T5, Canada

Tel +I 2042723 I 40

Fax + I 8777768332

Email shawn.bugden@umanitoba.ca
Background: Citalopram is the most commonly prescribed antidepressant in Canada. Concerns have been raised about its cardiac safety, and a dose-dependent prolongation of the QT interval has been documented. Drug interactions involving concomitant use of other medications that prolong the QT interval or increase citalopram levels by interfering with its metabolism increase the cardiac risk. Regulatory bodies (Health Canada and the US Food and Drug Administration) issued warnings and required labeling changes in 2011/2012, suggesting maximum citalopram doses ( $<40 \mathrm{mg}$ for those $<65$ years; $<20 \mathrm{mg}$ for those $\geq 65$ years) and avoiding drug interactions that increase cardiac risk. The purpose of this study is to assess the impact of these warnings on citalopram prescribing practices.

Methods: A quasi-experimental interrupted time series analysis was conducted using all citalopram prescribing data from the population of Manitoba, Canada from 1999 to 2014. This allowed for the examination of high-dose prescribing (above regulatory warning levels) and the number of interacting medications per citalopram prescription.

Results: There was a dramatic decline in the prescribing of high doses in both age groups, with a $64.8 \%$ decline in those $<65$ years and $33.6 \%$ in those $\geq 65$ years. Segmented regression models indicated significant breakpoints in the third quarter of 2011 for both age groups $(P<0.0001)$, corresponding to the time the regulatory warnings were issued. There appeared to be no impact of the warnings on the prescribing of interacting medications. The number of interacting medications actually increased in the postwarning period $(<65,0.78-0.81$ interactions per citalopram prescription; $\geq 65,0.93-0.94, P<0.001)$.

Conclusion: Regulatory changes appear to have produced an important reduction in the highdose prescribing of citalopram. In contrast to this relatively simple dosage change, there was no indication that the more complex issue of resolving drug-drug interactions was impacted by regulatory warnings.

Keywords: citalopram, QT prolongation, safety warnings, interrupted time series

\section{Introduction}

Major depressive disorder is a relatively common, serious mental illness, with lifetime prevalence of $9.9 \% .{ }^{1}$ The most common pharmacological treatments for depression in Canada are the selective serotonin reuptake inhibitors (SSRIs), which were introduced to the Canadian marketplace in 1989. It was hoped that SSRIs would offer an equivalent efficacy but avoid the problems associated with older antidepressants: the sedative, anticholinergic side effects and cardiac toxicity of tricyclic antidepressants, and the strict dietary restrictions and the possible fatal drug interactions for monoamine oxidase inhibitors. The SSRI citalopram (Celexa ${ }^{\circledR}$, Forest Laboratories, Inc., New York, NY, USA) was introduced to the Canadian market in 1999 and has risen to become 
the most commonly prescribed antidepressant in Canada. Citalopram is now the 11 th most commonly prescribed drug of any class, with an estimated 7,302,000 prescriptions filled in Canada in 2012. ${ }^{2}$

Unfortunately, postmarketing assessment has raised some concerns about the cardiac safety of citalopram. There is an increasing body of evidence showing that citalopram interferes with heart conduction and prolongs the QT interval in a dose-dependent way. ${ }^{3} \mathrm{QT}$ interval prolongation is associated with an increased risk of torsade de pointes (TdP), a life threatening cardiac arrhythmia and sudden cardiac death. Postmarketing evidence includes case reports, pharmacovigilance studies, and pharmacoepidemiological studies in a variety of countries, which have shown a relationship between higher doses of citalopram and QT prolongation, TdP, and sudden cardiac death. ${ }^{3-7}$ In addition, studies looking at several SSRIs together have found that this is not a class effect, and that citalopram differs significantly from the rest of the drugs in this class. ${ }^{8,9}$

Realization of the possible cardiac effects of citalopram has led to increased focus on its potential drug interactions. Citalopram has a long half-life ( $~ 37$ hours), allowing for once daily dosing. It undergoes extensive hepatic metabolism, primarily by CYP3A4 and CYP2C19, and to a lesser extent by CYP2D6. ${ }^{10}$ Any medication that inhibits these enzymes will decrease citalopram metabolism, increasing the potential cardiac risk because of the dose-dependent impact on the QT interval. In addition, there are a large number of other medications that are known to prolong the QT interval. Concomitant use of multiple medications that prolong the QT interval is thought to have additive effects on the heart.

In response to these concerns, Health Canada issued an initial advisory in October 2011 acknowledging a potential link between citalopram, QT prolongation, and TdP. ${ }^{11}$ This was followed by a formal warning in January 2012 that included labeling changes for citalopram in regard to QT prolongation and the risk of TdP. The maximum recommended daily dose was lowered to $40 \mathrm{mg}$ for those under 65 years of age. A maximum of $20 \mathrm{mg}$ was recommended for those 65 years and older, those with hepatic impairment, and those taking drugs that inhibit CYP2C19. ${ }^{12}$ A similar warning process occurred in the United States with the US Food and Drug Administration (FDA) MedWatch communication and labeling changes in August 2011, and a revised recommendation in May 2012 (Figure 1). ${ }^{13}$

Historically, the impact of such advisories and warnings on the prescribing practices has been limited. ${ }^{14}$ One example of this limited response was the commonly prescribed gastrokinetic agent, cisapride (Propulsid ${ }^{\circledR}$, Janssen Pharmaceuticals, Inc., Titusville, NJ, USA). Cisapride also causes dose-related QT prolongation that is enhanced by drug interactions. In 1995, the FDA issued a black box warning for cisapride regarding the use of cisapride with interacting medications. A second warning was issued in 1998, expanding the list of contraindications to cisapride. Despite these warnings, the number of cardiac events associated with cisapride continued to increase, and use in persons with contraindications was essentially unchanged. ${ }^{15,16}$ Due to the ineffectiveness of educational interventions and warnings to limit the inappropriate use of cisapride, the drug manufacturer voluntarily withdrew cisapride from the market in 2000. Other medications have followed a similar path to withdrawal (terfenadine, astemizole, mibefradil). ${ }^{17}$ A systematic review of the impact of safety regulatory warnings found that only $41 \%$ of interrupted time series analyses reported an impact. ${ }^{14}$ To the best of our knowledge, there has been no previous major population assessment of the impact of the regulatory warnings on citalopram prescribing.

In this study, we examined the impact of regulatory body intervention (ie, public warnings, labeling changes) on the prescribing patterns of citalopram, with a quasi-experimental

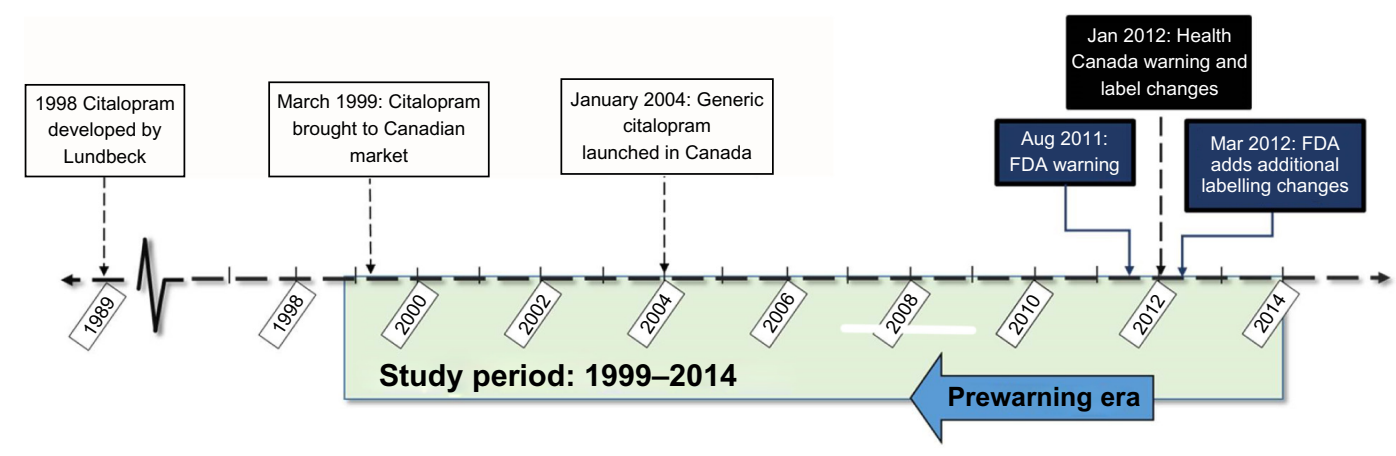

Figure I Citalopram regulatory timeline.

Abbreviation: FDA, US Food and Drug Administration. 
interrupted time series analysis using real-world population level administrative data.

\section{Methods}

In a cohort of all users of citalopram aged 18 or older, we examined citalopram dosage and use of interacting medications over a 15-year period (April 1, 1999 to March 31, 2014) in Manitoba, Canada (population 1.2 million). This time frame includes the interval within which the Health Canada and FDA warnings were issued. Data were obtained from the Drug Program Information Network (DPIN) through the Manitoba Centre for Health Policy. The DPIN is a centralized system used to process outpatient prescriptions and claims to the universal provincial medication insurance plan (Pharmacare) and third-party insurers. It contains records for almost every outpatient prescription dispensed in Manitoba. Approval for this study was given by the University of Manitoba Health Research Ethics Board and the Manitoba Health Information Privacy Commission.

The daily dose of citalopram was calculated for each prescription based on tablet strength, quantity dispensed, and duration of the prescription. Records with null values in the quantity dispensed field were removed as part of the data cleaning process. Daily doses for such prescriptions are not calculable, but due to their small number $(n=77)$ they would not be expected to impact the study results. In cases where individuals received two prescriptions of different strengths of citalopram on the same date, the dose was interpreted to be the sum of the dispensations, and a single derived prescription replaced the two original records. We classified citalopram prescription as either high dose or appropriate dose. High dose was defined as exceeding the January 2012 Health Canada advisory maximum daily dose of $40 \mathrm{mg} /$ day for persons less than 65 , and over $20 \mathrm{mg} /$ day for persons 65 years of age and over. The percentage of all citalopram prescriptions within each fiscal quarter that were high dose was then calculated by age group.

We constructed our interaction list using two primary data sources: the QTDrugs List ${ }^{\circledR}$ (including drugs with a "Known Risk" or "Possible Risk" of TdP) $)^{18}$ and the Lexi-Drugs ${ }^{\circledR}$ drug information system interactions (including " $\mathrm{X}$ - avoid combination" or "D - consider therapy modification"). ${ }^{19}$ The amalgamated list included all medications that either caused QT prolongation or produced a metabolic interaction resulting in decreased citalopram clearance. Concurrent prescriptions for other drugs were counted if they were found on this list of interacting drugs and if the duration of the prescription overlapped an interval covered by a citalopram prescription.
Segmented regression analysis was used to examine the effect that the Health Canada and FDA warnings had on high-dose prescribing. The primary outcome considered was the proportion of citalopram prescriptions above the recommended maximum dose before and after the Health Canada warning. This outcome was chosen as an outcome to avoid the issue of seasonality typically seen in antidepressant usage. ${ }^{20}$ As a secondary outcome, we compared the mean number of interacting medications associated with each citalopram prescription before and after the Health Canada warnings were issued.

All analyses were done using SAS 9.4 ${ }^{\circledR}$ (SAS Institute Inc., Cary, NC, USA).

\section{Results}

Between 1999 and the end of March 2014, there were 2,587,741 citalopram prescriptions dispensed in Manitoba. These were dispensed to 130,748 unique persons, providing just over 219,000 patient-years of antidepressant therapy. Of these, $78.2 \%(102,279)$ were under the age of 65 . The remaining $21.8 \%(28,469)$ were 65 years of age or older. The number of citalopram users rose continuously throughout the entire study period in both age groups (Figure 2). The mean dose prescribed differed significantly between age groups. Persons under 65 were prescribed a mean daily dose of $29.66 \mathrm{mg}$ (95\% confidence interval [CI], 29.6429.69), compared with $21.50 \mathrm{mg}$ (95\% CI, 21.25-21.75) for those 65 and older, a difference of $8.17 \mathrm{mg}$ (95\% CI, 7.91-8.42).

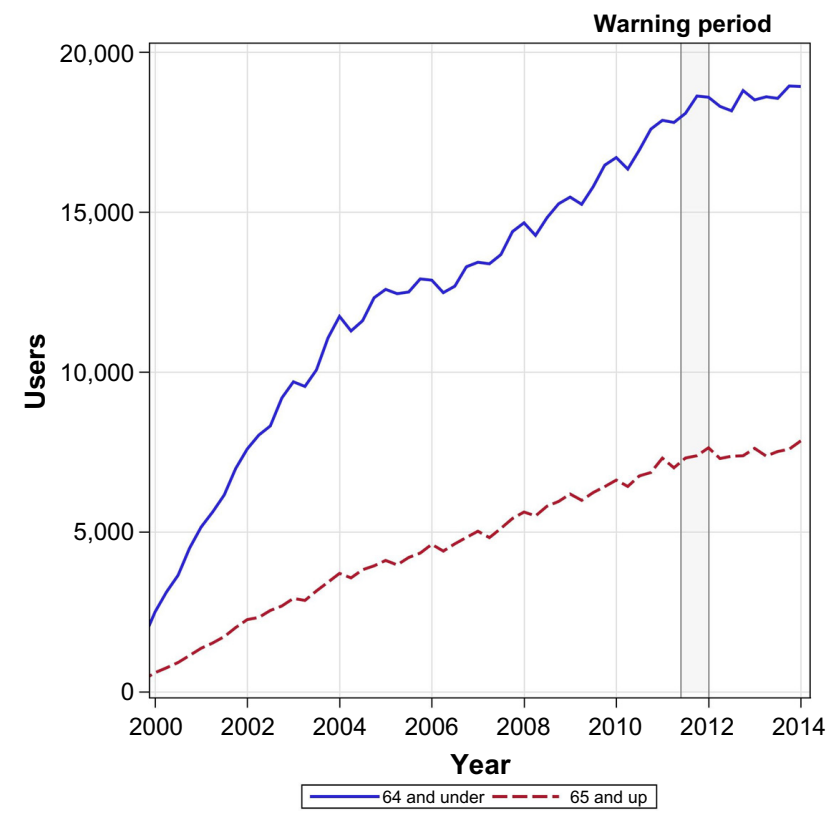

Figure 2 Number of citalopram users by age group. 
The proportion of citalopram prescriptions that were high dose is depicted in Figures 3 and 4. There was a modest rise in the percentage of high-dose citalopram prescriptions in both age groups until the warnings about higher dosages were issued in 2012. There is a clear drop in the percentage of high-dose citalopram prescriptions in 2012, immediately following the Health Canada warnings. In persons 65 years of age or older, the high-dose use dropped by $33.6 \%$, from a high of $28.3 \%$ immediately prior to the warning, to $18.8 \%$ by 2014. The drop in high-dose use in those less than 65 years was more pronounced at $64.8 \%$, from a high of $14.5 \%$ to a low of $5.1 \%$ after the warning.

We further assessed the impact of the Health Canada warning on prescribing with segmented regression models. The model for the $<65$ age group provided a good fit to the data and was highly significant $\left(\mathrm{F}_{3,26}=346.34, P<0.0001\right)$, with a significant breakpoint in the third quarter of 2011 $(P<0.001)$ (Figure 3). Similarly, the model for the $\geq 65$ age group was also significant $\left(\mathrm{F}_{3,24}=80.91, P<0.0001\right)$, again with a breakpoint in the third quarter of $2011(P<0.001)$ (Figure 4). The abrupt change in the direction of the trend, from increasing or plateauing to a sharp drop, argues in favor of an effect of the dose warnings on the prescribing patterns of citalopram.

The mean number of interacting drugs per citalopram prescription increased steadily over the study period from 0.61 interactions per prescription in 2000 to 0.87 interactions

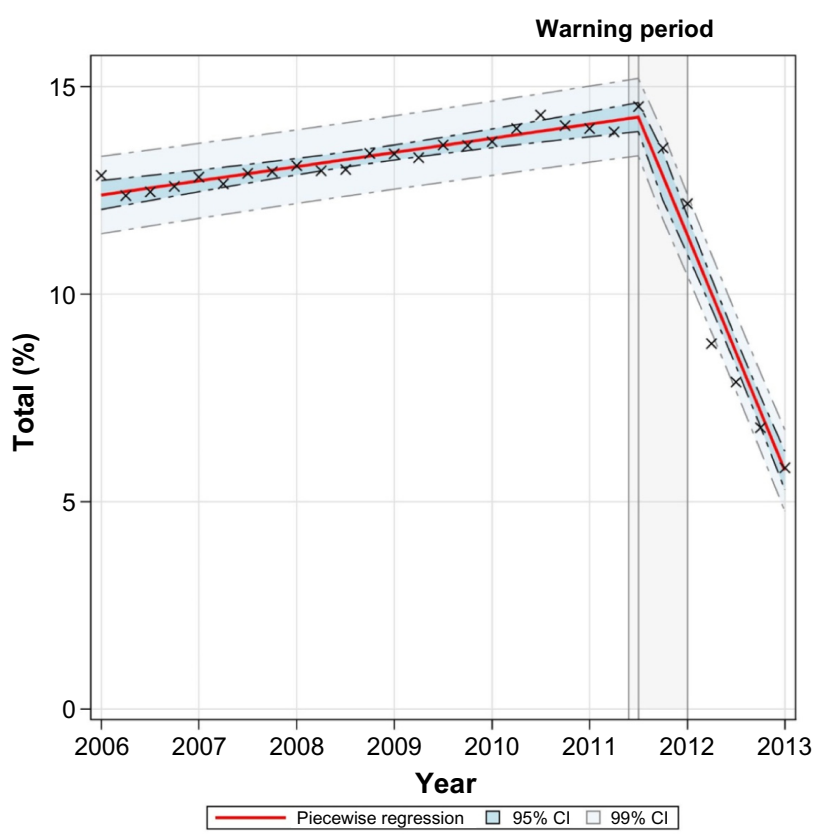

Figure 3 Segmented regression of percentage of high-dose citalopram prescriptions ( $<65$ age group).

Abbreviation: $\mathrm{Cl}$, confidence interval.

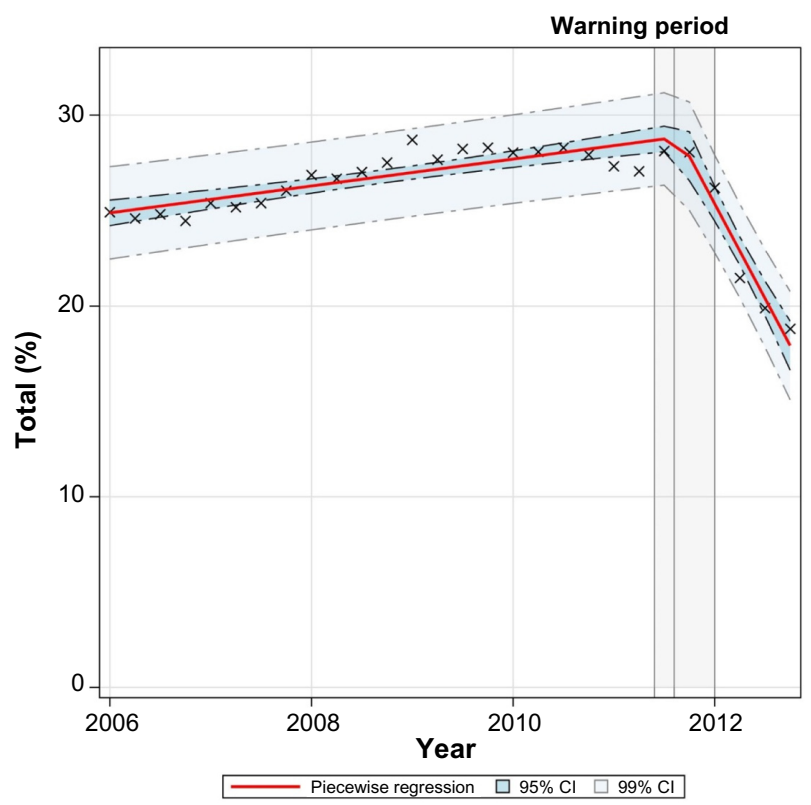

Figure 4 Segmented regression of percentage of high-dose citalopram prescriptions ( $\geq 65$ age group).

Abbreviation: $\mathrm{Cl}$, confidence interval.

per prescription in 2013 (Figure 5). There was no apparent impact of the warnings on the prescribing of interacting drugs, and segmented regression analysis failed to reveal any breakpoint in the data. An examination of the number of interactions before and after the warnings showed a small but statistically significant increase in the number of interactions per citalopram prescription in both age groups (Figure 6).

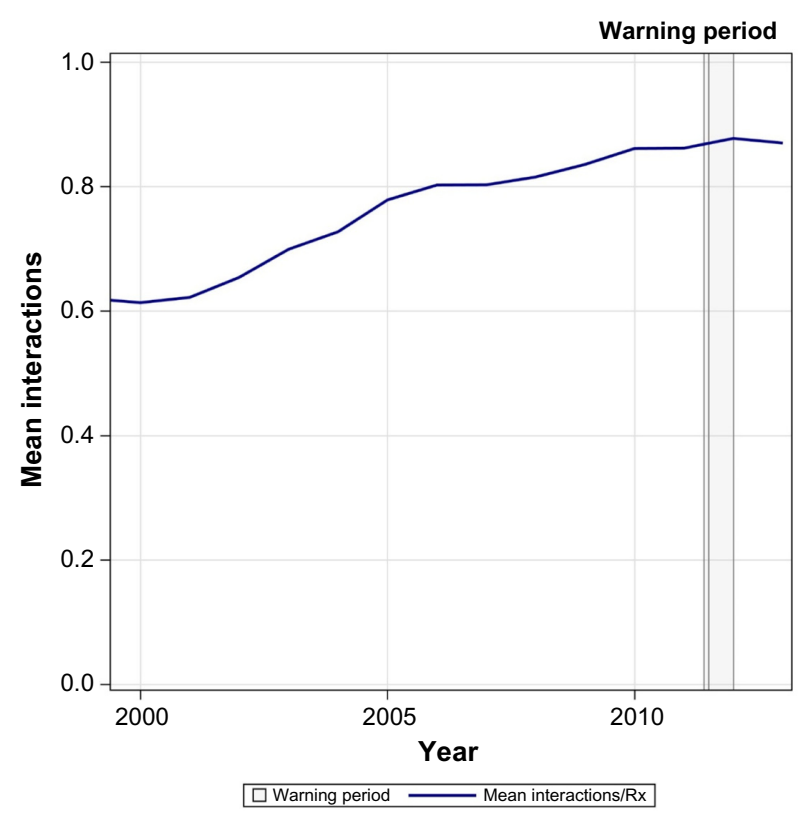

Figure 5 Number of interacting drugs per citalopram prescription. Abbreviation: $\mathrm{Rx}$, prescription. 


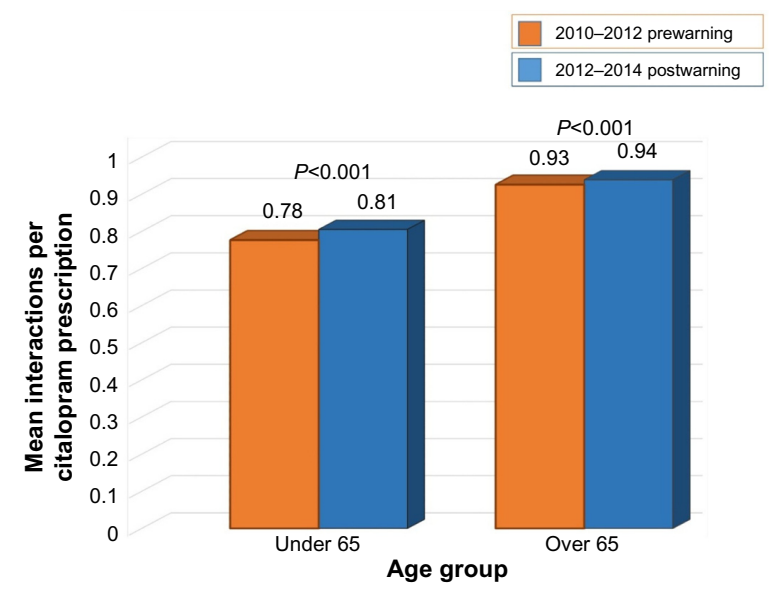

Figure 6 Citalopram interactions before and after regulatory warnings.

\section{Discussion}

Despite the lack of response that has been seen with some previous regulatory warnings related to QT prolongation, there was a substantial reduction in the proportion of highdose prescriptions following warnings regarding citalopram (Figures 3 and 4). For those under age of 65, high-dose prescriptions fell to $5.1 \%$ of total prescriptions. While a decline was also seen for those 65 years and older, the absolute level of high-dose prescribing remains substantial in this group at $18.8 \%$ of citalopram prescriptions. Given that cardiac events generally and QT prolongation specifically occur more frequently in older adults, ${ }^{21}$ this level of high-dose prescribing remains a concern. The higher risk for older adults is the reason that the warnings dictate a lower maximum dose $(20 \mathrm{mg})$ for those 65 years and older. Therefore, although lower average doses $(21.50 \mathrm{mg}$ for $\geq 65 ; 29.66 \mathrm{mg}$ for $<65$ ) were used in older adults, a greater proportion of the citalopram prescriptions were above the recommended maximum dose. A similar pattern of results was seen in a small study from England which also showed significant changes in citalopram prescribing following the FDA warnings, but a much smaller change in persons over the age of $60 .{ }^{22}$

The number of citalopram users continued to grow throughout the study period (Figure 2), including the period after the regulatory warnings. The series of warnings from late 2011 to early mid 2012 coincides with the observed decline in the percentage of high-dose citalopram prescriptions. Segmented regression analysis suggested the breakpoint for the decline near the end of 2011 in the midst of the warning period (Figure 1). There appears to be an increased sensitivity to safety issues currently than seen in the past, and an appropriate and sizeable change in prescribing practice in response to the relatively passive educational intervention of warnings and labeling changes. However, a reduction of dosage is a relatively simple intervention, and this may have facilitated the robust response.

The regulatory warnings included information on both dosage and drug interactions. Unfortunately, we found no evidence of a response to the citalopram warning related to interactions. In fact, the number of interactions per citalopram prescription actually increased in the postwarning periods. Responding to warnings regarding interactions is more complicated than simply decreasing the dose. Such changes may involve multiple prescribers, and clinicians are known to struggle with the clinical relevance of drug interactions and suffer from alert fatigue related to such issues. ${ }^{23,24}$ It would appear that despite the good intentions seen in reducing citalopram dosages, the response to the regulatory warning runs into barriers when the required action is less straightforward. A study of 333 patients from one English center also failed to show that prescribers adequately addressed the issue of medications interacting with citalopram in the postwarning period. $^{22}$

The study had a number of limitations. Citalopram use in hospitals was not included in the study. However, given that depression is primarily treated on an outpatient basis, the study captures the vast majority of citalopram use in Manitoba. The study captures population level use in Manitoba. There is no reason to expect that Manitoba would be different than other Canadian provinces, but caution should be taken in generalizing these results to other jurisdictions. The study also failed to directly assess the impact of high-dose prescribing on QT prolongation or its associated detrimental cardiac outcomes.

Not all available data supports the clinical relevance of the FDA/Health Canada warnings. ${ }^{24}$ Some pharmacoepidemiological data have failed to find a significant increase in the risk of negative cardiac outcomes compared to other SSRIs. ${ }^{25}$ This subtext may limit the response to these warnings by some clinicians. Furthermore, depending on patient history and response to therapy, continued high-dose citalopram therapy may be appropriate in selected patients. ${ }^{26,27}$ Individual assessment of benefit and risk is necessary, and zero highdose citalopram therapy may not be a realistic goal.

Overall, the results are encouraging for the safety of patients with a demonstrated dramatic change in prescribing patterns in response to a regulatory warning. Citalopram appears to provide a positive example of an appropriate response to a regulatory warning. There do, however, appear to be limitations to the response. The complexity of dealing with drug interactions seems to have limited the safety 
response of clinicians. This may reflect the reality of the limitations of the processes we have for dealing with drug interactions. Passive educational regulatory drug warnings may facilitate simple prescribing changes, but more complex changes are likely to require more robust interventions and support measures to ensure safe practice.

\section{Acknowledgments}

This work was supported by a University of Manitoba Centre on Aging Fellowship to SB.

The authors acknowledge the Manitoba Centre for Health Policy for approval to use the data contained in the Population Health Research Data Repository under project \#2013/2014-53. The results and conclusions are those of the authors and no official endorsement by the Manitoba Centre for Health Policy, Manitoba Health, or other data providers is intended or should be inferred. Data used in this study are from the Population Health Research Data Repository housed at the Manitoba Centre for Health Policy, University of Manitoba and were derived from data provided by Manitoba Health.

\section{Disclosure}

The authors report no conflicts of interest in this work.

\section{References}

1. Patten SB, Williams JVA, Lavorato DH, Wang JL, McDonald K, Bulloch AGM. Descriptive epidemiology of major depressive disorder in Canada in 2012. Can J Psychiatry. 2015;60(1):23-30.

2. TOP 100 DRUGS 2012. Pharm Pract (Granada). 2013;29(1):37-41. Available from: http://www.canadianhealthcarenetwork.ca/pharmacists/ news/special-reports/top-100-drugs-19660. Accessed August 13, 2015.

3. FDA.gov [homepage on the Internet]. FDA Drug Safety Communication: revised recommendations for Celexa (citalopram hydrobromide) related to a potential risk of abnormal heart rhythms with high doses. [updated February 15, 2013]. Available from: http://www.fda.gov/Drugs/DrugSafety/ucm297391.htm. Accessed May 20, 2015.

4. Åkström-Lilja C, Odeberg JM, Ekman E, Hägg S. Drug-induced torsades de pointes: a review of the Swedish pharmacovigilance database. Pharmacoepidemiol Drug Saf. 2008;17(6):587-592.

5. Deshmukh A, Ulveling K, Alla V, Abuissa H, Airey K. Prolonged QTc interval and torsades de pointes induced by citalopram. Tex Heart Inst $J$. 2012;39(1):68-70.

6. Drye LT, Spragg D, Devanand DP, et al. Changes in QTc interval in the Citalopram for agitation in Alzheimer's disease (CitAD) randomized trial. PLoS One. 2014;9(6):e98426.

7. Niemeijer MN, van den Berg ME, Hofman A, Jan A. Use of selective serotonin reuptake inhibitors and the heart-rate corrected QT interval in a real-life setting: the population-based Rotterdam Study. Br J Clin Pharmacol. Epub May 13, 2015

8. Funk KA, Bostwick JR. A comparison of the risk of QT prolongation among SSRIs. Ann Pharmacother. 2013;47(10):1330-1341.
9. Beach SR, Kostis WJ, Celano CM, et al. Meta-analysis of selective serotonin reuptake inhibitor-associated QTc prolongation. $J$ Clin Psychiatry. 2014;75(5):441-449.

10. Celexa ${ }^{\circledR}$. Compendium of Pharmaceuticals and Specialties. Toronto: Canadian Pharmacists Association; 2015.

11. Healthycanadians.gc.ca [homepage on the Internet]. Citalopram: Health Canada reviewing dose-related heart risk - report a concern. [updated January 31, 2013]. Available from: http://www.healthycanadians. gc.ca/recall-alert-rappel-avis/hc-sc/2011/13549a-eng.php. Accessed May 22, 2015.

12. Healthycanadians.gc.ca [homepage on the Internet]. Celexa (citalopram)-association with abnormal heart rhythms [updated March 4, 2013]. Available from: http://www.healthycanadians.gc.ca/recall-alertrappel-avis/hc-sc/2012/16887a-eng.php. Accessed May 22, 2015.

13. FDA.gov [homepage on the Internet]. FDA Drug Safety Communication: abnormal heart rhythms associated with high doses of Celexa (citalopram hydrobromide) [updated March 3, 2012]. Available from: http://www.fda.gov/Drugs/DrugSafety/ucm269086.htm. Accessed May 20, 2015.

14. Piening S, Haaijer-Ruskap FM, de Vries JTN, et al. Impact of safetyrelated regulatory action on clinical practice: a systematic review. Drug Saf. 2012;35(5):373-385.

15. Peña AS. Post-marketing reports of QT prolongation and ventricular arrhythmia in association with cisapride and Food and Drug Administration regulatory actions. Am J Gastroenterol. 2001;96(6):1698-1703.

16. Smalley W, Shatin D, Wysowski DK, et al. Contraindicated use of cisapride: impact of food and drug administration regulatory action. JAMA. 2000;284(23):3036-3039.

17. Woosley RL. Drug labeling revisions - guaranteed to fail? JAMA. 2000;284(23):3047-3049.

18. Woosley R, Romero K. QTdrugs list [web-based database]. [updated May 27, 2015]. Available from: https://www.crediblemeds.org. Accessed June 11, 2015.

19. online.lexi.com [homepage on the Internet]. Citalopram monograph: interactions. Lexi-Drugs-Lexicomp. Available from: http://online.lexi. com. Accessed June 11, 2015.

20. Gardarsdottir H, Egberts TCG, van Dijk L, Heerdink ER. Seasonal patterns of initiating antidepressant therapy in general practice in the Netherlands during 2002-2007. J Affect Disord. 2010; 122(3):208-212.

21. Trinkley KE, Page RL, Lien H, Yamanouye K, Tisdale JE. QT interval prolongation and the risk of torsades de pointes: essentials for clinicians. Curr Med Res Opin. 2013;29(12):1719-1726.

22. Austin J, Yi K, Agius M, Zaman R. The impact of guidance on citalopram's effects on the QT period on the practice of clinicians. Psychiatr Danub. 2014;26:226-230.

23. Ko Y, Abarca J, Malone DC, et al. Practitioners' views on computerized drug-drug interaction alerts in the VA system. J Am Med Inform Assoc. 2007;14(1):56-64.

24. Payne TH, Hines LE, Chan RC, et al. Recommendations to improve the usability of drug-drug interaction clinical decision support alerts. J Am Med Inform Assoc. 2015;(520):1-10.

25. Zivin K, Pfeiffer PN, Bohnert ASB, et al. Evaluation of the FDA warning against prescribing citalopram at doses exceeding $40 \mathrm{mg}$. Am J Psychiatry. 2013;170(6):642-650.

26. Howland RH. A critical evaluation of the cardiac toxicity of citalopram: part 2. J Psychosoc Nurs Ment Health Serv. 2011;49(12):13-16.

27. Howland RH. A critical evaluation of the cardiac toxicity of citalopram: part 1. J Psychosoc Nurs Ment Health Serv. 2011;49(11):13-16. 
Drug, Healthcare and Patient Safety

Dovepress

\section{Publish your work in this journal}

Drug, Healthcare and Patient Safety is an international, peer-reviewed open-access journal exploring patient safety issues in the healthcare continuum from diagnostic and screening interventions through to treatment, drug therapy and surgery. The journal is characterized by the rapid reporting of reviews, original research, clinical, epidemiological and

post-marketing surveillance studies, risk management, health literacy and educational programs across all areas of healthcare delivery. The manuscript management system is completely online and includes a very quick and fair peer-review system. Visit http://www.dovepress.com/ testimonials.php to read real quotes from published authors.

Submit your manuscript here: http://www.dovepress.com/drug-healthcare-and-patient-safety-journal 